5 Fr diagnostic catheters with the arteriotomy of a traditional 4Fr sheath. This is achieved by decreasing the wall thickness of the sheath thereby making it much softer and more likely to kink. We report our experience using this for femoral angiography.

Methods We retrospectively reviewed a prospectively-maintained IRB-approved institutional database of the senior authors to identify all cases where the 5/4RS was utilized. Case details were recorded including types of catheters used, incidence of kinking, closure technique, and length of lying flat (LOF). Technical success was defined as femoral access that allowed catheterization of all desired vessels with a $5 \mathrm{Fr}$ diagnostic catheter.

Results Over the study period, 50 consecutive femoral access diagnostic angiography cases were identified where the 5/4RS was utilized. A 5Fr JB1 glide catheter (Terumo) was utilized in all 50 cases and a SIM2 (Terumo) was utilized in 12 (24\%). Kinking of the 5/4RS occurred in $5(10 \%)$ patients, which was managed by restoring the lumen with the $5 \mathrm{Fr}$ diagnostic catheter, did not preclude technical success. Each of the incidents of kinking occurred in patients with BMI $>30$. Exovascular non-dilating groin closure (Mynx, Cardinal) was performed in $32(64 \%)$ cases and manual compression in 18 (36\%). All patients were discharged from recovery with 4 hours of total flat time with no symptomatic groin hematomas.

Conclusion The safety of trans-femoral cerebral angiography can be enhanced using a $4 \mathrm{Fr}$ groin access with a radial $5 / 4$ slender sheath with the known benefits of utilizing $5 \mathrm{Fr}$ diagnostic catheters.

Disclosures J. Campos: None. Z. Hsu: None. D. Zarrin: None. K. Golshani: None. N. Beaty: 2; C; Medtronic Neurovascular, Stryker Neurovascular. M. Bender: None. G. Colby: 2; C; Medtronic Neurovascular, MicroVention-Terumo, Stryker Neurovascular. L. Lin: 2; C; Medtronic Neurovascular, MicroVention-Terumo, Stryker Neurovascular. A. Coon: 2; C; Avail MedSystems, Imperative Care, InNeuroCo, Medtronic Neurovascular, MicroVention-Terumo, Q'apel, Rapid Medical, Stryker Neurovascular.

\section{E-028 IMMEDIATE PROCEDURAL OUTCOMES OF HIGH MESH- DENSITY FLOW DIVERTER PLACEMENT IN THE UPPER BASILAR ARTERY/BASILAR APEX FOR THE TREATMENT OF CEREBRAL ANEURYSMS: EXPERIENCE WITH 15 CONSECUTIVE SURPASS ${ }^{\mathrm{TM}}$ FLOW DIVERSION CASES}

1J Campos*, ${ }^{1} \mathrm{Z}$ Hsu, ${ }^{2} \mathrm{D}$ Zarrin, ${ }^{1} \mathrm{~K}$ Golshani, ${ }^{3} \mathrm{~N}$ Beaty, ${ }^{4} \mathrm{M}$ Bender, ${ }^{2} \mathrm{G}$ Colby, ${ }^{5} \mathrm{~L}$ Lin, ${ }^{5} \mathrm{~A}$ Coon. ${ }^{1}$ Department of Neurological Surgery, University of California, Irvine, Orange, $C A$; ${ }^{2}$ Department of Neurosurgery, University of California, Los Angeles, Los Angeles, CA; ${ }^{3}$ Department of Neurosurgery, Tallahassee Neurological Clinic, Tallahassee, FL; ${ }^{4}$ Department of Neurosurgery, University of Rochester Medical Center, Rochester, NY; ${ }^{5}$ Department of Neurosurgery, Carondelet Neurological Institute, St. Joseph's Hospital, Tucson, AZ

\subsection{6/neurintsurg-2021-SNIS. 124}

Background Recent studies have demonstrated acceptable safety and efficacy of posterior circulation flow diversion for cerebral aneurysms. However, these studies have overwhelmingly included V4 segment and proximal basilar aneurysms (range 70-90\%) and analyzed 48-wire devices (Pipeline, Silk, etc.) that have lower mesh densities. The Surpass flow diverter (Fremont, CA) with its 64, 72, and 96-strand designs is a high-mesh density device that is known for its increased flow diversion and perforator preservation properties. We present here the first known dedicated series of upper basilar and basilar apex flow diverter cases using the Surpass device.

Methods We retrospectively reviewed a prospectively-maintained IRB-approved institutional database of the senior authors to identify all cases where a Surpass ${ }^{\mathrm{TM}}$ flow diverter (Streamline or Evolve) was implanted in the upper basilar or basilar apex. Case details were recorded including patient demographics, aneurysm type, device used, and periprocedural events and complications. Technical success was defined as successful implantation of the device without intraprocedural device removal.

Results Over the 20-month study period, 15 cases of Surpass flow diversion (9 (60\%) Evolve, 6 (40\%) Streamline) cases were performed where a device was placed in the upper basilar artery/basilar apex with the basilar apex being crossed in 11 cases. The aneurysms treated included 5 (33\%) basilar apex, 5 (33\%) superior cerebellar basilar, and 5 (33\%) basilar trunk aneurysms. Dual antiplatelet therapy was established with ticagrelor $90 \mathrm{mg}$ BID and aspirin $81 \mathrm{mg}$ QD in each patient. Intraprocedural tirofiban bolus (1/2 cardiac dose) and subsequent drip was employed in 11 (73\%) of cases regardless. Technical success was achieved in $100 \%$ of cases and there were no instances of in-stent platelet aggregation. No major strokes were observed in the first 30 days after each case, and all patients were discharged at their MRS baseline.

Conclusion The treatment of upper basilar and basilar apex aneurysms with the high mesh-density Surpass can be safely performed. Further studies are needed to evaluate occlusion rates and efficacy as well as the applicability of these results to lower mesh-density 48-wire implants.

Disclosures J. Campos: None. Z. Hsu: None. D. Zarrin: None. K. Golshani: None. N. Beaty: 2; C; Medtronic Neurovascular, Stryker Neurovascular. M. Bender: None. G. Colby: 2; C; Stryker Neurovascular. 6; C; Proctor for Stryker Neurovascular, MicroVention-Terumo, and Medtronic Neurovascular. L. Lin: 2; C; Medtronic Neurovascular, Stryker Neurovascular, MicroVention-Terumo. 6; C; Proctor for Medtronic Neurovascular, Stryker Neurovascular, MicroVention-Terumo. A. Coon: 2; C; Avail MedSystems, Imperative Care, InNeuroCo, Medtronic Neurovascular, MicroVention-Terumo, Q'apel, Rapid Medical, Stryker Neurovascular.

\section{E-029 RESCUE INTRACRANIAL ANGIOPLASTY WITH OR WITHOUT STENTING IN ACUTE ISCHEMIC STROKE}

${ }^{1} \mathrm{~K}$ Khatibi*, ${ }^{2} \mathrm{~A}$ Mowla, ${ }^{1} \mathrm{H}$ Saber, ${ }^{1} \mathrm{~L}$ Ponce Mejia, ${ }^{1} \mathrm{~N}$ Kaneko, ${ }^{1} \mathrm{G}$ Duckwiler, ${ }^{1} \mathrm{~S}$ Tateshima. ${ }^{1}$ Radiology, UCLA, Los Angeles, CA; ${ }^{2}$ Neurosurgery, USC, Los Angeles, CA

10.1136/neurintsurg-2021-SNIS. 125

Introduction The appropriate management of acute ischemic strokes secondary to hemodynamic intracranial atherosclerotic disease refractory to the conventional mechanical thrombectomy remains unclear. We aimed to investigate the clinical outcome of the patients who underwent rescue intracranial angioplasty with or without stenting in the setting of acute ischemic stroke at our institution.

Method This is a retrospective single-arm observational study to evaluate the effect of acute rescue angioplasty with or without stenting on clinical symptom burden and functional outcome. We included all patients that underwent such rescue intervention within 7 days of presenting with acute ischemic stroke with large vessel occlusion between the years of 2017 